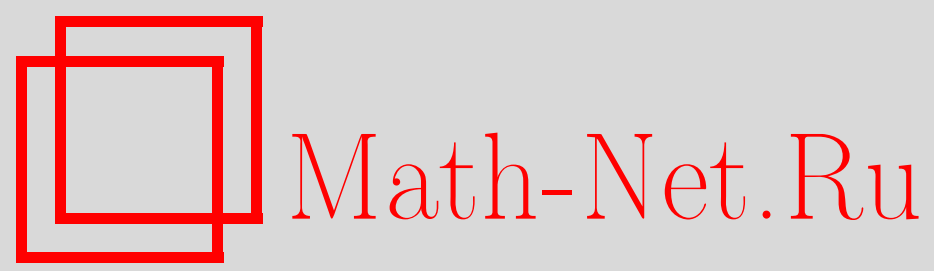

Д. Х. Казанчян, В. М. Круглов, Условие равномерной интегрируемости экспоненциальных мартингалов, Вестник ТвГУ. Серия: Прикладная математика, 2020, выпуск 3, 5-13

DOI: https://doi.org/10.26456/vtpmk596

Использование Общероссийского математического портала Math-Net.Ru подразумевает, что вы прочитали и согласны с пользовательским соглашением

http: //www. mathnet.ru/rus/agreement

Параметры загрузки:

IP: 3.80 .253 .173

26 апреля 2023 г., 15:33:51 


\section{ТЕОРИЯ ВЕРОЯТНОСТЕЙ И МАТЕМАТИЧЕСКАЯ СТАТИСТИКА}

\section{УДК 519.2 \\ УСЛОВИЕ РАВНОМЕРНОЙ ИНТЕГРИРУЕМОСТИ ЭКСПОНЕНЦИАЛЬНЫХ МАРТИНГАЛОВ}

Казанчян Д.Х., Круглов В.М.

МГУ им. М.В. Ломоносова, г. Москва

Поступила в редакиию 08.08.2020, после переработки 12.10.2020.

В статье доказано новое достаточное условие для равномерной интегрируемости экспоненциальных локальных мартингалов. Условие сформулировано в терминах квадратической вариации локального мартингала. Оно обобщает ряд известных достаточных условий подобного рода.

Ключевые слова: равномерная интегрируемость, экспоненциальные процессы, мартингалы, марковские моменты.

Вестник ТвГУ. Серия: Прикладная математика. 2020. № 3. С. 5-13. https://doi.org/10.26456/vtpmk596

\section{1. Введение}

На протяжении всей статьи используется традиционная терминология, принятая в теории мартингалов, см., например, учебник [2]. Пусть даны вероятностное пространство $(\Omega, \mathcal{F}, \mathbb{F})$ и фильтрация $\mathbb{F}=\left\{\mathcal{F}_{t}: \mathcal{F} \subseteq \mathcal{F}, t \geq 0\right\}$, удовлетворяющие обычным условиям. Пусть непрерывный локальный мартингал $M=\left\{M_{t}, t \geq 0\right\}, M_{0}=0$, относительно фильтрации $\mathbb{F}$ определен на вероятностном пространстве $(\Omega, \mathcal{F}, \mathbb{P})$. Известно (см. [2], глава 4$)$, что существует непрерывный возрастающий процесс $[M]=\left\{[M]_{t}, t \geq 0\right\},[M]_{0}=0$, согласованный с фильтрацией, $\mathbb{F}$ такой, что разность $M^{2}-[M]=\left\{M_{t}^{2}-[M]_{t}, t \geq 0\right\}$ является локальным мартингалом. Случайный процесс $[M]$ называется квадратической вариацией локального мартингала $M$. Известно, что для любого вещественного числа $\alpha$ случайный процесс $\alpha M=\left\{\alpha M_{t}, t \geq 0\right\}$ снова является локальным мартингалом, и случайные процессы $[\alpha M]$ и $\alpha^{2}[\bar{M}]$ являются неотличимыми. Последнее обозначает, что существует множество $\Omega^{\prime} \in \mathcal{F}, \mathbb{P}\left\{\Omega^{\prime}\right\}=1$, такое, что $[\alpha M]_{t}(\omega)=\alpha^{2}[M]_{t}(\omega)$ для всех $t \geq 0$ и для всех $\omega \in \Omega^{\prime}$. Для любого марковского момента $\tau$ относительно фильтрации $\mathbb{F}$ можно определить новый случайный процесс $M^{\tau}=\left\{M_{t \wedge \tau}, t \geq 0\right\}$, называемый остановленным процессом в марковский момент $\tau$. Здесь и далее используется обозначение $a \wedge b=\min \{a, b\}$ для любых вещественных чисел $a$ и $b$. Если $M$ является непрерывным локальным мартингалом относительно фильтрации $\mathbb{F}$, то $M^{\tau}$ также является непрерывным локальным мартингалом относительно фильтрации $\mathbb{F}$. Нетрудно доказать, что для любого марковского момента $\tau$ случайные процессы $\left[M^{\tau}\right]$ и $[M]^{\tau}$ неотличимы. 
Понятие локального мартингала зависит от вероятностного пространства $(\Omega, \mathcal{F}, \mathbb{P})$ и фильтрации $\mathbb{F}$. Они считаются фиксированными. Также предполагается, что все рассматриваемые далее случайные процессы согласованы с фильтрацией $\mathbb{F}$ и все марковские моменты определены относительно этой фильтрации. По этой причине мы не будем всякий раз напоминать о вероятностном пространстве и фильтрации.

В книге [2] (см. стр. 120) можно найти доказательство, что для любого непрерывного локального мартингала $M, M_{0}=0$, и для любого вещественного числа $\alpha$ экспоненциальный процесс $e^{\alpha M-\alpha^{2}[M] / 2}$ также является непрерывным локальным мартингалом. Можно доказать (см., например, [8], стр. 101), что положительный локальный мартингал является супермартингалом. Поэтому выполняется следующие неравенства

$$
\mathbb{E} e^{\alpha M_{t}-\alpha^{2}[M]_{t} / 2} \leq \mathbb{E} e^{\alpha M_{0}-\alpha^{2}[M]_{0} / 2}=1 \text { для всех } t \geq 0 .
$$

Известная важная проблема (см., например, книгу [4]) состоит в отыскании условий для равномерной интегрируемости экспоненциального случайного процесса $e^{M-[M] / 2}$. Ряд условий равномерной интегрируемости таких случайных процессов можно найти в [1], [3], [4], [6], [7], [9], [9], [10].

Напомним, что дан непрерывный локальный мартингал $M=\left\{M_{t}, t \geq 0\right\}$ такой, что $M_{0}=0, \lim _{t \rightarrow \infty} M_{t}=M_{\infty},\left|M_{\infty}\right|<\infty$ п.в., $[M]_{\infty}<\infty$ п.в. Известно, что равномерная интегрируемость экспоненциального процесса $e^{M_{t}-[M]_{t} / 2}$ равносильна равенству $\mathbb{E} e^{M_{\infty}-[M]_{\infty} / 2}=1$. Простое доказательство этого утверждения можно найти в статье [5].

Только, что было установлено, что для любого вещественного числа $\alpha$ экспоненциальный процесс $e^{\alpha M-\alpha^{2}[M] / 2}=\left\{e^{\alpha M_{t}-\alpha^{2}[M]_{t} / 2}, t \geq 0\right\}$ является равномерно интегрируемым, если и только, если

$$
\mathbb{E} e^{\alpha M_{\infty}-\alpha^{2}[M]_{\infty} / 2}=1
$$

В частности, это равенство выполняется для $\alpha=1$, если выполняются условия:

$$
\begin{array}{ll}
{[M]_{\infty} \leq \text { const. }} & \text { Гирсанов }(1960), \\
\mathbb{E} e^{[M]_{\infty} / 2}<\infty & \text { Новиков }(1972), \\
\sup _{t \geq 0} \mathbb{E} e^{M_{t} / 2}<\infty & \text { Kazamaki }(1977), \\
\mathbb{E} e^{[M]_{\infty} / 2-c \sqrt{[M]_{\infty}}<\infty, 0<c-\text { число }} & \text { Новиков }(1979), \\
\liminf _{\varepsilon \downarrow 0}\left(\mathbb{E} e^{(1-\varepsilon)[M]_{\infty} / 2}\right)^{\varepsilon}<\infty & \text { Крылов }(1995), \\
\liminf _{\varepsilon \downarrow 0}\left(\sup _{t \geq 0} \mathbb{E} e^{(1-\varepsilon) M_{t} / 2}\right)^{\varepsilon}<\infty & \text { Крылов }(1995) .
\end{array}
$$

Специалистам известно, что условие Kazamaki строго слабее условия Новикова(1972). Условие Крылова $\liminf _{\varepsilon \downarrow 0}\left(\sup _{t \geq 0} \mathbb{E} e^{(1-\varepsilon) M_{t} / 2}\right)^{\varepsilon}<\infty$ строго слабее условия Kazamaki. Очевидно, что условие Новикова (1979) строго слабее условия Новикова (1972).

В этой статье мы усиливаем утверждение Новикова (1979). 
Теорема 1. Равенство (2) выполнятся для $0<\alpha \leq 1$, если

$$
\liminf _{\varepsilon \downarrow 0}\left(\mathbb{E} e^{[M]_{\infty} / 2-c \sqrt{(1+\varepsilon)[M]_{\infty}}}\right)^{\varepsilon}<\infty
$$

для некоторого числа $c>0$.

С помощью примера будет показано, что условие (3) строго слабее условия Новикова (1979).

\section{2. Доказательство}

Нам потребуется утверждение Новикова (1972). Оригинальное доказательство довольно трудное. Простое доказательство утверждения Новикова (1972) можно найти в статье [5]. Сначала будет доказано равенство (2) для $0<\alpha<1$ при условии (3). Из условия (3) следует, что существуют положительные числа $0<\varepsilon_{m}<1, m=1,2 \ldots$, такие, что $\varepsilon_{m} \downarrow 0$ при $m \uparrow \infty$ и

$$
\mathbb{E} e^{[M]_{\infty}-c \sqrt{\left(1+\varepsilon_{m}\right)[M]_{\infty}}}<\infty, \lim _{m \rightarrow \infty}\left(\mathbb{E} e^{[M]_{\infty}-c \sqrt{\left(1+\varepsilon_{m}\right)[M]_{\infty}}}\right)^{\varepsilon_{m}}=d<\infty
$$

Для данного числа $\varepsilon_{m}$ найдется число $\lambda=\lambda\left(\alpha, \varepsilon_{m}, c\right)>0$ такое, что на множестве $\left\{[M]_{\infty}>\lambda\right\}$. будет выполняться неравенство $\alpha^{2}[M]_{\infty} \leq[M]_{\infty}-c \sqrt{\left(1+\varepsilon_{m}\right)[M]_{\infty}}$. Отсюда следует, что

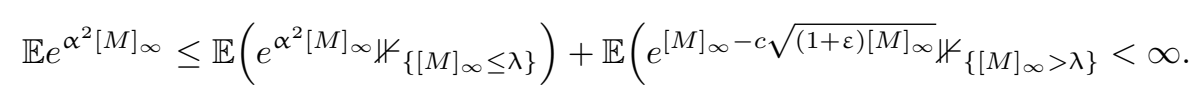

Можно применить утверждение Новикова (1972) к локальному мартингалу $\alpha M=\left\{\alpha M_{t}, t \geq 0\right\}$ с квадратичной вариацией $[\alpha M]=\left\{\alpha^{2}[M]_{t}, t \geq 0\right\}$. Тем самым неравенство (2) доказано для $0<\alpha<1$.

Снова предположим, что $0<\alpha<1$. Для фиксированного $\varepsilon_{m}$ мы определим марковский момент

$$
\tau=\tau\left(\varepsilon_{m}, c\right)=\inf \left\{t \geq 0: M_{t} \leq[M]_{t}-c \sqrt{\left(1+\varepsilon_{m}\right)[M]_{t}}-\varepsilon_{m}^{-2}\right\}, \text { где } \inf \{\emptyset\}=\infty .
$$

Так как экспоненциальный локальный мартингал $e^{\alpha_{m} M-\alpha_{m}^{2}[M] / 2}$ равномерно интегрируем, то выполняется равенство

$$
\mathbb{E} e^{\alpha_{m} M_{\tau}-\alpha_{m}^{2}[M]_{\tau} / 2}=1
$$

В силу того, что случайные процессы $M$ и $[M]$ непрерывны, выполняется равенство

$$
M_{\tau}=[M]_{\tau}-c \sqrt{\left(1+\varepsilon_{m}\right)[M]_{\tau}}-\varepsilon_{m}^{-2} \text { на множестве }\{\tau<\infty\} .
$$

Из определения марковского момента $\tau$ следует, что

$$
M_{t}>[M]_{t}-c \sqrt{\left(1+\varepsilon_{m}\right)[M]_{t}}-\varepsilon_{m}^{-2} \text { на множестве }\{\tau=\infty\} \text { для всех } t \geq 0 .
$$

Полагая здесь $t \rightarrow \infty$, мы получим неравенство

$$
M_{\infty} \geq[M]_{\infty}-c \sqrt{\left(1+\varepsilon_{m}\right)[M]_{\infty}}-\varepsilon_{m}^{-2} \text { п.в. на множестве }\{\tau=\infty\} .
$$


Это неравенство вместе предыдущим равенством приводят к следующему неравенству

$$
M_{\tau} \geq[M]_{\tau}-c \sqrt{\left(1+\varepsilon_{m}\right)[M]_{\tau}}-\varepsilon_{m}^{-2} \text { п.в. }
$$

$\mathrm{C}$ помощью этого неравенства мы получим для всех $0<\alpha<1$, что

$$
\begin{aligned}
e^{\alpha M_{\tau}-\alpha^{2}[M]_{\tau} / 2} & =e^{M_{\tau}-[M]_{\tau} / 2} e^{(\alpha-1) M_{\tau}+\left(1-\alpha^{2}\right)[M]_{\tau} / 2} \\
& \leq e^{M_{\tau}-[M]_{\tau} / 2} e^{-(1-\alpha)^{2}[M]_{\tau} / 2+c(1-\alpha) \sqrt{\left(1+\varepsilon_{m}\right)[M]_{\tau}}+\epsilon_{m}^{-2}} \\
& =e^{M_{\tau}-[M]_{\tau} / 2} e^{-\left((1-\alpha) \sqrt{[M]_{\tau}}-c \sqrt{1+\varepsilon_{m}}\right)^{2} / 2+c^{2}\left(1+\varepsilon_{m}\right) / 2+\varepsilon_{m}^{-2}} \\
& \leq e^{M_{\tau}-[M]_{\tau} / 2} e^{c^{2}\left(1+\varepsilon_{m}\right) / 2+\varepsilon_{m}^{-2}} .
\end{aligned}
$$

Последняя в этой цепочке случайная величина интегрируема, так как $\mathbb{E} e^{M_{\tau}-[M]_{\tau} / 2} \leq 1$, как будет показано ниже. По теореме об ограниченной сходимости мы получим, что

$$
\lim _{\alpha \uparrow 1} \mathbb{E}\left|e^{\alpha M_{\tau}-\alpha^{2}[M]_{\tau} / 2}-e^{M_{\tau}-[M]_{\tau} / 2}\right|=0 .
$$

Отсюда, в свою очередь, следует

$$
1=\lim _{\alpha \uparrow 1} \mathbb{E} e^{\alpha M_{\tau}-\alpha^{2}[M]_{\tau} / 2}=\mathbb{E} e^{M_{\tau}-[M]_{\tau} / 2} .
$$

Принимая во внимание (5), мы получим

$$
\begin{gathered}
1=\mathbb{E} e^{M_{\tau}-[M]_{\tau} / 2}=\mathbb{E}\left(e^{M_{\tau}-[M]_{\tau} / 2}\left(\nVdash_{\{\tau=\infty\}}+\nVdash_{\{\tau<\infty\}}\right)\right) \leq \\
\leq \mathbb{E} e^{M_{\infty}-[M]_{\infty} / 2}+\mathbb{E}\left(e^{M_{\tau}-[M]_{\tau} / 2} \nVdash_{\{\tau<\infty\}}\right), \\
\mathbb{E}\left(e^{M_{\tau}-[M]_{\tau} / 2} \nVdash_{\{\tau<\infty\}}\right) \leq\left(\mathbb{E}\left(e^{M_{\tau}-[M]_{\tau} / 2} \nVdash_{\{\tau<\infty\}}\right)^{\varepsilon_{m}}=\right. \\
=\left(\mathbb{E}\left(e^{[M]_{\tau} / 2-c \sqrt{\left(1+\varepsilon_{m}\right)[M]_{\tau}}-\varepsilon_{m}^{-2} \nVdash_{\{\tau<\infty\}}}\right)^{\varepsilon_{m}} .\right.
\end{gathered}
$$

Последняя величина стремится к нулю при $m \rightarrow \infty$ по условию (3) и, следовательно, $\mathbb{E} e^{M_{\infty}-[M]_{\infty} / 2}=1$. Действительно, функция $x / 2-c \sqrt{\left(1+\varepsilon_{m}\right) x}, x \geq c^{2}\left(1+\varepsilon_{m}\right)$, возрастает и, следовательно,

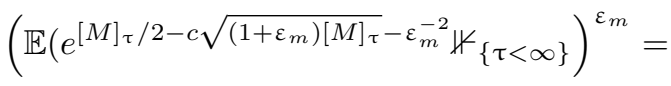

$$
\begin{aligned}
& =\left(\mathbb{E} e^{[M]_{\tau} / 2-c \sqrt{\left(1+\varepsilon_{m}\right)[M]_{\tau}}-\varepsilon_{m}^{-2}}\left(\nVdash_{\left\{[M]_{\tau}<c^{2}\left(1+\varepsilon_{m}\right)\right\}}+\nVdash_{\{M]_{\tau} \geq c^{2}\left(1+\varepsilon_{m}\right\}}\right)\right)^{\varepsilon_{m}} \leq \\
& \leq\left(\mathbb{E} e^{c^{2}\left(1+\varepsilon_{m}\right)-\varepsilon_{m}^{-2}} \nVdash_{\left\{[M]_{\tau}<c^{2}\left(1+\varepsilon_{m}\right)\right\}}+\mathbb{E} e^{[M]_{\tau} / 2-c \sqrt{\left(1+\varepsilon_{m}\right)[M]_{\tau}}-\varepsilon_{m}^{-2} \nVdash_{\left\{[M]_{\tau} \geq c^{2}\left(1+\varepsilon_{m}\right\}\right.}}\right)^{\varepsilon_{m}} \\
& \leq\left(e^{c^{2}\left(1+\varepsilon_{m}\right)-\varepsilon_{m}^{-2}}+\mathbb{E} e^{[M]_{\infty} / 2-c \sqrt{\left(1+\varepsilon_{m}\right)[M]_{\infty}}-\varepsilon_{m}^{-2}}\right)^{\varepsilon_{m}} \rightarrow 0 \text { при } m \rightarrow \infty .
\end{aligned}
$$

Нам осталось доказать неравенство $\mathbb{E} e^{M_{\tau}-[M]_{\tau} / 2} \leq 1$. Выше отмечалось, что случайный процесс $M^{\tau}=\left\{M_{\tau \wedge t}, t \geq 0\right\}$ является непрерывным локальным мартингалом. В силу (1) выполняется неравенство $\mathbb{E} e^{M_{\tau \wedge t}-[M]_{\tau \wedge t} / 2} \leq 1$ для всех $t \geq 0$. 
Применив лемму Фату, мы получим требуемое неравенство $\mathbb{E} e^{M_{\tau}-[M]_{\tau} / 2} \leq 1$. Теорема доказана.

Теперь мы построим пример непрерывного локального мартингала, для которого выполнено условие (3), но условие Новикова (1979) не выполняется.

Пример 1. Предположим, что непрерывный процесс броуновского движения $B=\left\{B_{t}, t \geq 0\right\}$ и случайная величина $\tau$ с плотностью вероятностей $p(x)=b e^{-x / 2+c \sqrt{x} \nVdash_{[0, \infty)}}(x)$ определены на вероятностном пространстве $(\Omega, \mathcal{F}, \mathbb{P})$ и независимы; здесь $c>0$ - число и $1 / b=\int_{0}^{\infty} e^{-x / 2+c \sqrt{x}} d x$. Нетрудно доказать, что случайный процесс $M=\left\{M_{t}, t \geq 0\right\}, M_{t}=B_{t \wedge \tau}$, является мартингалом относительно фильтрации $\mathbb{G}=\left\{G_{t}, t \geq 0\right\}, G_{t}=\sigma\left(\tau, B_{s}, 0 \leq s \leq t\right)$. Определим новую фильтрацию $\mathbb{F}=\left\{\mathcal{F}_{t}, t \geq 0\right\}$ по правилу $\mathcal{F}_{t}=\cap_{s>t} \sigma\left(\mathcal{G}_{s}, \mathcal{N}\right)$, где $\mathcal{N}$ обозначает класс событий $A \in \mathcal{F}, \mathbb{P}\{A\}=0$. Легко видеть, что так построенная фильтрация $\mathbb{F}$ удовлетворяет обычным условиям. Очевидно, что случайный процесс $M=\left\{M_{t}, t \geq 0\right\}, M_{t}=B_{t \wedge \tau}$, является непрерывным локальным мартингалом относительно фильтрации $\mathbb{F}$.

Убедимся, что $[M]_{t}=t \wedge \tau$. Разобьем сегмент $[0, t], t>0$, точками $t_{n, k}=k 2^{-n} t, k=0, \ldots, 2^{n}$. Простые вычисления показывают, что

$$
\begin{aligned}
& \mathbb{E}\left|\sum_{k=1}^{2^{n}}\right| B_{t_{n, k}}-\left.B_{t_{n, k-1}}\right|^{2}-\left.t\right|^{2}=\mathbb{E}\left|\sum_{k=1}^{2^{n}}\left(\left|B_{t_{n, k}}-B_{t_{n, k-1}}\right|^{2}-\left(t_{n, k}-t_{n, k-1}\right)\right)\right|^{2}=\frac{t^{2}}{2^{n-1}} \\
& \sum_{n=1}^{\infty} \mathbb{E}\left|\sum_{k=1}^{2^{n}}\right| B_{t_{n, k}}-\left.B_{t_{n, k-1}}\right|^{2}-\left.t\right|^{2}=\sum_{n=1}^{\infty} \frac{t^{2}}{2^{n-1}}=2 t^{2}
\end{aligned}
$$

Отсюда следует, что

$$
\lim _{n \rightarrow \infty} \sum_{k=1}^{2^{n}}\left|B_{t_{n, k}}-B_{t_{n, k-1}}\right|^{2}=t \text { п.в. }
$$

По определению квадратической вариации $\left[B^{\tau}\right]=\left\{\left[B^{\tau}\right]_{t}, t \geq 0\right\}$ (см. [2], стр. 76) мы получим

$$
\begin{aligned}
{\left[B^{\tau}\right]_{t} } & =\lim _{n \rightarrow \infty} \sum_{k=1}^{2^{n}}\left|B_{t_{n, k} \wedge \tau}-B_{t_{n, k-1} \wedge \tau}\right|^{2}= \\
& =\lim _{n \rightarrow \infty} \sum_{k=1}^{2^{n}}\left|B_{t_{n, k}}-B_{t_{n, k-1}}\right|^{2}=t \text { п.в. на множестве }\{t \leq \tau\} . \\
{\left[B^{\tau}\right]_{t} } & =\lim _{n \rightarrow \infty} \sum_{k=1}^{2^{n}}\left|B_{t_{n, k} \wedge \tau}-B_{t_{n, k-1} \wedge \tau}\right|^{2}= \\
& =\lim _{n \rightarrow \infty} \sum_{k: t_{n, k} \leq \tau}\left|B_{t_{n, k}}-B_{t_{n, k-1}}\right|^{2}=\tau \text { п.в. на множестве }\{\tau<t\} .
\end{aligned}
$$

Таким образом доказано требуемое равенство $\left[B^{\tau}\right]_{t}=t \wedge \tau$ п.п. для всех $t \geq 0$.

Локальный мартингал $M=B^{\tau}$ не удовлетворяет условию Новикова (1979), так как $[M]_{\infty}=\tau$ и $\mathbb{E} e^{\tau / 2-c \sqrt{\tau}}=\infty$. С другой стороны, оно удовлетворяет условию (3). 
Действительно,

$$
\begin{gathered}
\lim _{\varepsilon \downarrow 0}\left(\mathbb{E} e^{\tau / 2-c \sqrt{(1+\varepsilon) \tau}}\right)^{\varepsilon}=\lim _{\varepsilon \downarrow 0}\left(b \int_{0}^{\infty} e^{-c(\sqrt{(1+\varepsilon}-1) \sqrt{x}} d x\right)^{\varepsilon}= \\
=\lim _{\varepsilon \downarrow 0}\left(\frac{2 b(\sqrt{1+\varepsilon}+1)^{2}}{c^{2} \varepsilon}\right)^{\varepsilon}=1 .
\end{gathered}
$$

\section{Заключение}

В статье доказано новое достаточное условие для равномерной интегрируемости непрерывных экспоненциальных локальных мартингалов. Показано, что предложенное достаточное условие строго слабее ряда известных достаточных условий для равномерной интегрируемости экспоненциальных локальных мартингалов.

\section{Список литературы}

[1] Cherny A., Shiryaev A. On criteria for the uniform integrability of Brownian stochastic exponentials // Optimal Control and Partial Differential Equations. Eds. by J.L. Menaldi et al.. Iso Press, 2001. Pp. 1-13.

[2] Chung K.L., Williams R.J. Introduction to stochastic Integration. Second Edition. Boston: Birkhauser, 1990.

[3] Гирсанов И.В. О преобразовании одного класса случайных процессов с помощью абсолютно-непрерывной замены меры // Теория вероятностей и ее применения. 1960. Т. 5, № 3. С. 314-330.

[4] Kazamaki N. Continuous Exponential Martingales and BMO / eds. by A. Dold, B. Eckmann, F. Takens. Series: Lecture Notes in Mathematics. Vol. 1579. Berlin, Heidelberg, New York: Springer-Verlag, 1994. 91 p.

[5] Kazanchyan D.C., Kruglov V.M. Uniform integrability of exponential processes // Lobachevskii Journal of Mathematics. 2019. Vol. 40, № 10. Pp. 1498-1506. https://doi.org/10.1134/S1995080219100159

[6] Клебанера Ф.К., Липцер Р.Ш. Когда стохастическая экспонента является мартингалом. Развитие метода Бенеша // Теория вероятностей и ее применения. 2013. Т. 58, № 1. С. 53-81.

[7] Krylov N.V. Introduction to the theory of diffusion processes. Providence: American Mathematical Society, 1995.

[8] Medvegyev P. Stochastic Integration Theory. Oxford: Oxford University Press, 2007. $607 \mathrm{p}$.

[9] Новиков А.А. Об одном тождестве для стохастических интегралов // Теория вероятностей и ее применения. 1972. Т. 17, № 4. С. 761-765. 
[10] Новиков А.А. Об условиях равномерной интегрируемости непрерывных неотрицательных мартингалов // Теория вероятностей и ее применения. 1979. T. 24, № 4. C. 821-825.

\section{Образец цитирования}

Казанчян Д.Х., Круглов В.М. Условие равномерной интегрируемости экспоненциальных мартингалов // Вестник ТвГУ. Серия: Прикладная математика. 2020. № 3. C. 5-13. https://doi.org/10.26456/vtpmk596

\section{Сведения об авторах}

\section{1. Казанчян Драстамат Хачатурович}

аспирант кафедры математической статистики факультета ВМиК МГУ им. М.В. Ломоносова.

Россия, 119992, г. Москва, ГСП-1, Ленинские горы, МГУ.

E-mail:drastamat94@gmail.com

\section{2. Круглов Виктор Макарович}

профессор кафедры математической статистики факультета ВМиК МГУ им. М.В. Ломоносова.

Россия, 119992, г. Москва, ГСП-1, Ленинские горы, МГУ. 


\title{
A NEW SUFFICIENT CONDITION FOR UNIFORM INTEGRABILITY OF EXPONENTIAL LOCAL MARTINGALES
}

\author{
Kazanchyan Drastamat Chachaturovich \\ PhD student of Mathematical Statistics department, \\ Faculty of Computational Mathematics and Cybernetics, \\ Lomonosov Moscow State University \\ Russia, 119992, Moscow, GSP-1, 1-52, Leninskiye Gory, MSU. \\ E-mail:drastamat94@gmail.com \\ Kruglov Victor Makarovich \\ Professor of Mathematical Statistics department, \\ Faculty of Computational Mathematics and Cybernetics, \\ Lomonosov Moscow State University \\ Russia, 119992, Moscow, GSP-1, 1-52, Leninskiye Gory, MSU.
}

Received 08.08.2020, revised 12.10.2020.

In the article it is suggested a new sufficient condition for uniform integrability of continues exponential local martingales. It is shown that the condition is much weaker then a number of known sufficient conditions for uniform integrability of exponential local martingales.

Keywords: uniform integrability, exponential processes, martingales, stopping times, martingales.

\section{Citation}

Kazanchyan D.Ch., Kruglov V.M., "A new sufficient condition for uniform integrability of exponential local martingales", Vestnik TvGU. Seriya: Prikladnaya Matematika [Herald of Tver State University. Series: Applied Mathematics], 2020, № 3, 5-13(in Russian). https://doi.org/10.26456/vtpmk596

\section{References}

[1] Cherny A., Shiryaev A., "On criteria for the uniform integrability of Brownian stochastic exponentials", Optimal Control and Partial Differential Equations, eds. J.L. Menaldi et al., Iso Press, 2001, 1-13.

[2] Chung K.L., Williams R.J., Introduction to stochastic Integration, Second Edition, Birkhauser, Boston, 1990.

[3] Girsanov I.V., "On Transforming a Certain Class of Stochastic Processes by Absolutely Continuous Substitution of Measures", Theory of Probability and its Applications, 5:3 (1960), 314-330 (in Russian).

[4] Kazamaki N., Continuous Exponential Martingales and BMO. V.1579, Lecture Notes in Mathematics, eds. A. Dold, B. Eckmann, F. Takens, Springer-Verlag, Berlin, Heidelberg, New York, 1994, 91 pp. 
[5] Kazanchyan D.C., Kruglov V.M., "Uniform integrability of exponential processes", Lobachevskii Journal of Mathematics, 40:10 (2019), 1498-1506, https://doi.org/10.1134/S1995080219100159.

[6] Klebaner F., Liptser R., "When a stochastic exponential is a true martingale. Extention of method of Bene's", Theory of Probability and its Applications, 58:1 (2014), 38-62.

[7] Krylov N.V., Introduction to the theory of diffusion processes, American Mathematical Society, Providence, 1995.

[8] Medvegyev P., Stochastic Integration Theory, Oxford University Press, Oxford, 2007, 607 pp.

[9] Novikov A.A., "On an identity for stochastic integrals", Theory of Probability and its Applications, 17:4 (1973), 717-720.

[10] Novikov A.A., "On the conditions of the uniform integrability of the continuous nonnegative martingales", Theory of Probability and its Applications, 24:4 (1980), $820-824$. 